\title{
FDG PET/CT for the diagnosis and management of infective endocarditis: Expert consensus vs evidence-based practice
}

\author{
Wengen Chen, MD, PhD, ${ }^{a}$ and Vasken Dilsizian, MD, MASNC ${ }^{\mathrm{a}}$ \\ a Department of Diagnostic Radiology and Nuclear Medicine, University of Maryland School of \\ Medicine, Baltimore, MD
}

Received Jul 27, 2018; accepted Jul 27, 2018

doi: $10.1007 / \mathrm{s} 12350-018-1398-1$

\section{See related article, pp. 303-308}

Infective endocarditis can involve the native or prosthetic heart valves, intra-cardiac pacemaker lead or left ventricular assist device, and the diagnosis remains difficult and challenging. There has been a significant increase in the implantation of cardiac electronic device systems worldwide, and this growth has been accompanied by rises in device infections and hospital admissions. ${ }^{1}$ This is in part related to the aging population of these new device recipients, higher rate of comorbidities, and the emergence of drug-resistant bacteria. $^{2}$

Clinical diagnosis of infective endocarditis is based on the modified Duke criteria, which relies heavily on the diagnostic accuracy of echocardiography, either transthoracic (TTE) or transesophageal (TEE). ECG gated cardiac CT angiography (CTA) has been shown to have added value in providing high resolution images for visualization of complications of infective endocarditis such as pseudo-aneurysms, fistulas, valve perforation, abscess, valvular dehiscence, and vegetation. Thus, TEE and CTA are currently the first line imaging choices for the diagnosis of both native and device-related infective endocarditis. However, TEE may miss $30 \%$ of infective endocarditis, ${ }^{3}$ and findings of TEE and CTA are often non-specific and are associated

Reprint requests: Vasken Dilsizian, MD, MASNC, Department of Diagnostic Radiology and Nuclear Medicine, University of Maryland School of Medicine, 22 S. Greene Street, Room N2W78, Baltimore, MD 21201-1595; vdilsizian@umm.edu

J Nucl Cardiol 2019;26:313-5.

$1071-3581 / \$ 34.00$

Copyright (C) 2018 American Society of Nuclear Cardiology. with significant artifacts, such as streaking and beam hardening artifact from the metal devices. More importantly, the establishment of infective endocarditis diagnosis is only the first step. It is critical to identify the extra-cardiac primary infection source as well as the spread of infection, such as infective embolism, in order to guide clinical management. Therefore, it would be desirable to have an imaging modality that not only identifies infective endocarditis but simultaneously assesses infection in extra-cardiac regions (primary culprit and/or embolic sources of infection).

\section{THE INCREMENTAL VALUE OF FDG PET/CT IN INFECTIVE ENDOCARDITIS}

${ }^{18}$ F-flurodeoxyglucose (FDG) positron emission tomography/computer tomography (PET/CT) provides functional molecular whole body imaging, which has been extremely useful for imaging various malignancies in oncology. Beyond identifying malignant cells, FDG uptake has been observed at sites of infection such as pneumonia, painful prosthetic joints, osteomyelitis, and complicated diabetic foot. The latter is a consequence of FDG targeting the inflammatory cells (macrophages, neutrophils, and lymphocytes) that overexpress the glucose transporters GLUT1 and GLUT4, and thereby accumulate with high concentration at the site of infection. Given the high spatial and target-to-background contrast resolution of FDG PET/CT, the technique can be applied for in vivo labeling of preexisting inflammatory cells in an infection site, ${ }^{4}$ and detect prosthetic valve endocarditis as well as cardiac implantable pacemaker and defibrillator infections.

Both the European Society of Cardiology (ESC) and the American Heart Association (AHA) guidelines are concordant in terms of indications for TEE and CTA in the assessment of patients with suspected infective endocarditis, however, there is discordance regarding 
the roles of FDG PET/CT between ESC and AHA. ${ }^{5}$ The ESC guideline states that FDG PET/CT may help reduce the number of misdiagnosed infective endocarditis classified in the "possible" category of the modified Duke criteria, and may help visualize peripheral emboli and metastatic infective events. ${ }^{6}$ On the other hand, the AHA guideline states that more studies are needed to determine the roles of FDG PET/CT in the diagnosis and management of patients with infective endocarditis. ${ }^{7}$

\section{WHY THE DISCREPANCY BETWEEN THE ESC AND AHA GUIDELINES?}

Unlike TEE and CTA, there are far less welldesigned prospective randomized studies with FDG $\mathrm{PET} / \mathrm{CT}$ to assess infective endocarditis. Evidence regarding the roles of FDG PET/CT is largely from observational case studies and retrospective data reviews. As such, the incremental value of FDG PET/ $\mathrm{CT}$ in cardiac implantable electronic devices and infective endocarditis is based on consensus or agreement among topic experts rather than based on the most robust scientific evidence. ${ }^{8}$ Such early adoption of a new technique in clinical care on the basis of recommendation from a panel of experts may improve patient outcomes, even if the scientific evidence is lacking.

FDG PET/CT has been used clinically for the diagnosis and management of patients with various infections for more than 2 decades. Despite the retrospective and observational nature of most studies, the published data for the evaluation of infection and particularly cardiac-device related infection are overall consistent and support its judicious application in the workup of infective endocarditis. ${ }^{8} 9$ FDG PET/CT may have unique roles over TEE and CTA in the following aspects: (1) to provide confirmatory information when TEE and CTA findings are inconclusive; (2) to diagnose infective endocarditis earlier than TEE and CTA before morphologic damage ensues; (3) to detect unexpected source of infection and embolism in the body; and (4) to potentially guide clinical management. ${ }^{10}$ It has been shown that treatment planning was changed in up to $35 \%$ of patients when FDG PET/CT was added to the endocarditis workup, including antibiotic treatment prolongation, referral to surgical procedures, and avoidance of unnecessary device extraction. ${ }^{11}$

For left ventricular assistant device (LVAD), although data are relatively limited, preliminary studies have shown that FDG PET/CT can differentiate and localize the site and extension of infection within the central portion of device or along the peripheral driveline, ${ }^{12}$ which has different clinical significances that, patients with infection involving the central portion of an LVAD (including the pump and cannula) have a poorer survival rate than those with an infection involving the peripheral driveline and exit site. ${ }^{13}$

\section{PROSTHETIC VS NATIVE VALVE ENDOCARDITIS}

For prosthetic valve endocarditis, a recent systemic review shows that FDG PET/CT has a sensitivity of 73$100 \%$, with a specificity of $71-100 \%$, positive predictive value of $67-100 \%$, and a negative predictive value of 50$100 \% .{ }^{14}$ It has been reported that adding FDG PET/CT to the Duke criteria increases its sensitivity from 52$70 \%$ to $91-97 \%$ without compromising specificity. ${ }^{15,16}$ However, it should be noted that for native valve infective endocarditis, current data are insufficient to support the role of FDG PET/CT. The sensitivity of FDG PET/CT has been reported to be $14 \%$ for native valve endocarditis. ${ }^{17}$ On the other hand, FDG PET/CT may influence clinical management of native valve endocarditis patients by identifying the primary extracardiac infection source or infective emboli. In a prospective study of patients with a diagnosed native valve infective endocarditis, FDG PET/CT identified additional infection sites in $74.5 \%$ of the patients, such as the lungs, skeleton, brain, and other organs. Based on the information of extra-cardiac findings on FDG PET/ $\mathrm{CT}$, the incidence of relapse infective endocarditis was decreased by 2 -folds because of appropriate intervention as a result of the extra-cardiac findings on FDG PET/ CT. ${ }^{18}$

\section{INFECTION VS INFLAMMATION}

A major concern of FDG PET/CT for infection diagnosis is its non-specific nature, as FDG can also be accumulated in neutrophils and macrophages in a sterile inflammation site. ${ }^{19}$ Thus, it is generally suggested that FDG PET/CT should not be performed within 3 months after surgical placement of a device, ${ }^{6}$ although other studies have shown no significant change of accuracy after surgery. ${ }^{15}$

FDG PET/CT study should be performed after a dietary preparation with a meal of high fat and low carbohydrates to suppress physiologic cardiac FDG uptake. Both attenuation corrected and non-attenuation corrected images should be evaluated and compared. In most cases, infection can be reasonably differentiated from non-infective inflammation with high accuracy based on the intensity of FDG uptake and more importantly the FDG distribution pattern around a device. A focal activity higher than the liver favors a true infection; while a mild diffuse activity surrounding a device is more likely an inflammatory change. Intensity alone such as standard uptake value (SUV) or target-to-background ratio (TBR) has not been shown to 
have a role in differentiating infection from inflammation. ${ }^{4}$ In cases where the differentiation between true infection and inflammation are difficult by FDG PET/ CT, a radiolabeled white blood cell scan can be performed, which is more specific for infection, but less sensitive compared to FDG PET/CT. ${ }^{20}$

\section{CONCLUSION}

Infective endocarditis, particularly device-related infective endocarditis, is associated with enormous societal/medical costs and mortality. Advanced imaging in early and accurate diagnosis of infective endocarditis is expected to significantly impact medical cost and more importantly, clinical outcome. Existing data justify the judicious use of FDG PET/CT in the workup of infective endocarditis. In addition, FDG PET/CT should be performed once infective endocarditis is diagnosed and proven clinically, for evaluation of infective embolism and primary extra-cardiac infection source. Centers for Medicare \& Medicaid Services registry may be an appropriate step for infective endocarditis evaluation by FDG PET/CT. With accumulation of more cases via the registry, the role of FDG PET/CT in assessing and managing infective endocarditis can then be verified.

\section{Disclosure}

Wengen Chen and Vasken Dilsizian have no conflicts of interest to declare.

\section{References}

1. Sajadi MM, Chen W, Dilsizian V. Targeted bacteria-specific ${ }^{18} \mathrm{~F}$ fluoro-maltohexaose but not fdg pet distinguishes infection from inflammation. JACC Cardiovasc Imaging 2018. https://doi.org/10. 1016/j.jcmg.2018.03.008.

2. Mind HG, Proclemer A. The 11th world survey of cardiac pacing and implantable cardioverter-defibrillators: calendar year 2009-a World Society of Arrhythmia's project. Pacing Clin Electrophysiol 2011;34:1013-27.

3. Hill EE, Herijgers P, Claus P, Vanderschueren S, Peetermans WE, Herregods MC. Abscess in infective endocarditis: the value of transesophageal echocardiography and outcome: A 5-year study. Am Heart J 2007;154:923-8.

4. Chen W, Kim J, Molchanova-Cook OP, Dilsizian V. The potential of FDG PET/CT for Early diagnosis of cardiac device and prosthetic valve infection before morphologic damages ensue. Curr Cardiol Rep 2014;16:459-66.

5. Murphy DJ, Din M, Hage FG, Reyes E. Guidelines in review: Comparison $\mathrm{f}$ ESC and AHA guidance for the diagnosis and management of infective endocarditis in adults. J Nucl Cardiol 2018. https://doi.org/10.1007/s12350-018-1333-5.
6. Habib G, Lancellotti P, Antunes MJ, et al. 2015 ESC Guidelines for the management of infective endocarditis: The Task Force for the Management of Infective Endocarditis of the European Society of Cardiology (ESC). Endorsed by: European Association for Cardio-Thoracic Surgery (EACTS), the European Association of Nuclear Medicine (EANM). Eur Heart J 2015;36:3075-128.

7. Baddour LM, Wilson WR, Bayer AS, et al. Infective endocarditis in adults: diagnosis, antimicrobial therapy, and management of complications: A scientific statement for healthcare professionals from the american heart association. Circulation 2015;132:143586.

8. Dilsizian V, Bacharach SL, Beanlands SR, et al. ASNC imaging guidelines/SNMMI procedure standard for positron emission tomography (PET) nuclear cardiology procedures. J Nucl Cardiol 2016;23:1187-226.

9. Dilsizian V, Achenbach S, Narula J. On adding versus selecting imaging modalities for incremental diagnosis: A case-study of ${ }^{18} \mathrm{~F}-$ fluorodeoxyglucose PET/CT in prosthetic valve endocarditis. JACC Cardiovasc Imaging 2013;6:1020-1.

10. Chen W, Sajadi MM, Dilsizian V. Merits of FDG PET/CT and functional molecular imaging over echocardiography and ct angiography for the diagnosis of cardiac device infections. JACC Cardiovasc Imaging (in press).

11. Orvin K, Goldberg E, Bernstine H, et al. The role of FDG-PET/CT imaging in early detection of extra-cardiac complications of infective endocarditis. Clin Microbiol Infect 2015;21:69-76.

12. Kim J, Feller ED, Chen W, Dilsizian V. FDG PET-CT imaging for LVAD associated infections. JACC Cardiovasc Imaging 2014;7:839-42. https://doi.org/10.1016/j.jcmg.2014.04.013

13. Kim J, Feller ED, Chen W, Liang Y, Dilsizian V. FDG PET/CT for early detection and localization of left ventricular assist device infection: Impact on patient management and outcome. JACC Cardiovasc Imaging 2018. https://doi.org/10.1016/j.jcmg.2018.01. 024.

14. Gomes A, Glaudemans AWJM, Touw DJ, et al. Diagnostic value of imaging in infective endocarditis: A systematic review. Lancet Infect Dis 2017;17:e1-14.

15. Saby L, Laas O, Habib G, et al. Positron emission tomography/computed tomography for diagnosis of prosthetic valve endocarditis: increased valvular ${ }^{18} \mathrm{~F}$-fluorodeoxyglucose uptake as a novel major criterion. J Am Coll Cardiol 2013;61:2374-82.

16. Pizzi MN, Roque A, Fernández-Hidalgo N, et al. Improving the diagnosis of infective endocarditis in prosthetic valves and intracardiac devices with ${ }^{18} \mathrm{~F}$-fluordeoxyglucose positron emission tomography/computed tomography angiography: Initial results at an infective endocarditis referral center. Circulation 2015;132:1113-26.

17. Salomäki SP, Saraste A, Kemppainen J, et al. ${ }^{18}$ F-FDG positron emission tomography/computed tomography in infective endocarditis. J Nucl Cardiol 2017;24:195-206.

18. Kestler M, Muñoz P, Rodríguez-Créixems M, et al. Role of (18)FFDG PET in Patients with infective endocarditis. J Nucl Med 2014;55:1093-8.

19. Dilsizian V, Chandrashekhar Y. Distinguishing active vasculitis from sterile inflammation and graft infection: A call for a more specific imaging target. JACC Cardiovasc Imaging 2017;10:10857.

20. Rouzet F, Chequer R, Benali K, et al. Respective performance of 18F-FDG PET and radiolabeled leukocyte scintigraphy for the diagnosis of prosthetic valve endocarditis. J Nucl Med 2014;55:1980-5. 